\title{
STABILITY ESTIMATES FOR A CLASS OF HELMHOLTZ PROBLEMS*
}

\author{
U. HETMANIUK ${ }^{\dagger}$
}

\begin{abstract}
This paper presents new stability estimates for the scalar Helmholtz equation with a complex-valued Robin boundary condition as well as Dirichlet and Neumann boundary conditions. For each estimate, we state the explicit dependency of constants on the wave number. To deal with mixed boundary conditions, we impose geometrical constraints on the two-dimensional or threedimensional bounded domain.
\end{abstract}

Key words. Helmholtz problems, stability estimates, mixed boundary conditions

AMS subject classifications. 35B35, 35J05

\section{Introduction}

The scalar Helmholtz equation defines the simplest model for wave phenomena in the frequency domain. Despite its simplicity, the mathematical analysis of this equation on a bounded domain remains incomplete. In particular, with a complexvalued Robin boundary condition, few stability estimates for the solution are available with the explicit dependency of the constants on the wave number. This dependence is important, for instance, in order to define relations between the wave number and the discretization parameters in the error analysis of a discretization method.

On one-dimensional bounded domains, Douglas et al. [5] proved such stability estimates for the Helmholtz equation with a Robin boundary condition. Ihlenburg and Babuška [10] analyzed the Helmholtz equation with mixed boundary conditions for one-dimensional domains. For two-dimensional and three-dimensional domains, stability estimates are available only for the Helmholtz equation with a Robin boundary condition. Melenk [11] proved optimal estimates in two dimensions with an original proof based on Rellich's identities [12]. Recently, Cummings and Feng [3] extended the proof of Melenk to three-dimensions ${ }^{1}$. However, the results of Melenk, Cummings, and Feng do not apply to Helmholtz problems with mixed boundary conditions.

Therefore, the goal of this paper is to fill this gap by presenting stability estimates for the Helmholtz equation with mixed boundary conditions. In Section 2, we introduce notations and the model problem. Section 3 states the geometric assumptions on the bounded domain and the stability estimates.

\section{Model problem}

2.1. Notations. Throughout this paper, we adopt the following notations.

- $\mathbb{R}$ is the set of real numbers.

- $\Omega$ denotes a bounded connected Lipschitz domain in $\mathbb{R}^{d}(d=2,3), \bar{\Omega}$ is the closure of $\Omega$, and $\partial \Omega$ the boundary of $\Omega$.

${ }^{*}$ Received: January 16, 2007; accepted (in revised version): June 14, 2007. Communicated by Claude LeBris.

${ }^{\dagger}$ Sandia National Laboratories, P.O. Box 5800, MS 1320, Albuquerque, NM 87185-1320, USA (ulhetma@sandia.gov). Sandia is a multiprogram laboratory operated by Sandia Corporation, a Lockheed Martin Company, for the United States Department of Energy under Contract DE-AC0494AL85000.

${ }^{1}$ The author proved concurrently an extension for a class of three-dimensional scattering problems in his PhD thesis [8]. 
- $\mathbf{v}$ denotes a vector and $\mathbf{x}$ the vector position in $\mathbb{R}^{d}(d=2,3) . \quad|\mathbf{v}|$ is the Euclidean norm of the vector $\mathbf{v}$ and $\mathbf{v} \cdot \mathbf{w}$ the Euclidean inner product of vectors $\mathbf{v}$ and $\mathbf{w}$.

- We denote $L^{2}(\Omega)=H^{0}(\Omega)$ to be the Sobolev space of square integrable complex-valued functions over $\Omega$ equipped with the norm

$$
\|v\|_{0, \Omega}=\sqrt{\int_{\Omega}|v(\mathbf{x})|^{2} d \mathbf{x} .}
$$

We use the notation $H^{s}(\Omega)$ for the Sobolev space of order $s$ in the usual sense. On $H^{s}(\Omega)$, the full norm is written $\|\cdot\|_{s, \Omega}$. For any positive integer $k$, we will also consider on $H^{k}(\Omega)$ the semi-norm

$$
|v|_{k, \Omega}=\left\|D^{k} v\right\|_{0, \Omega}
$$

- For a surface $\Gamma$ of $\mathbb{R}^{d}, L^{2}(\Gamma)$ is the Sobolev space of square integrable complexvalued functions over $\Gamma$ and $\|\cdot\|_{0, \Gamma}$ the usual norm for $L^{2}(\Gamma)$. Similarly, a hierarchy of Sobolev spaces $H^{s}(\Gamma)$, equipped with the norm $\|\cdot\|_{s, \Gamma}$, can be defined.

- When no confusion arises, we use $\int_{\Omega} f$ or $\int_{\Omega} f(\mathbf{x})$ to denote $\int_{\Omega} f(\mathbf{x}) d \mathbf{x}$.

- We denote the normal derivative operator in the direction of the vector $\boldsymbol{\nu}$ by $\partial_{\nu}$. When no confusion arises, we assume this vector to be outgoing.

- $\nabla$ is the gradient operator, div the divergence operator, and $\Delta$ the Laplacian operator.

2.2. Problem. We consider the Helmholtz equation defined over a bounded, connected Lipschitz domain $\Omega$ in $\mathbb{R}^{d}(d=2$ or 3$)$ :

$$
\left\{\begin{aligned}
-\Delta p-k^{2} p & =f & & \text { in } \Omega, \\
p & =0 & & \text { on } \Gamma_{d} \\
\partial_{\nu} p & =0 & & \text { on } \Gamma_{n} \\
\partial_{\nu} p & =(i \beta-\alpha) p+g & & \text { on } \Gamma_{r}
\end{aligned}\right.
$$

where the wave number $k$ is real and positive, $i$ denotes the imaginary unit, and the real-valued functions $\alpha$ and $\beta$ are bounded on $\Gamma_{r}$. The boundary of $\Omega$ is partitioned as follows

$$
\partial \Omega=\overline{\Gamma_{d} \cup \Gamma_{n} \cup \Gamma_{r}}
$$

where $\Gamma_{d}, \Gamma_{n}$, and $\Gamma_{r}$ are disjoint sets. We allow the cases where $\Gamma_{d}=\emptyset, \Gamma_{n}=\emptyset$, or $\Gamma_{d}=\Gamma_{n}=\emptyset$. However, we require that $\Gamma_{r}$ is non-empty, i.e. $\Gamma_{r}$ satisfies

$$
\operatorname{meas}\left(\Gamma_{r}\right)>0
$$

We assume that the source terms $f$ and $g$ belong respectively to $L^{2}(\Omega)$ and to $L^{2}\left(\Gamma_{r}\right)$. We define the functional space $H_{\Gamma_{d}}^{1}(\Omega)$ as

$$
H_{\Gamma_{d}}^{1}(\Omega)=\left\{v \in H^{1}(\Omega) ; v=0 \text { on } \Gamma_{d}\right\} ;
$$

we remark that when $\Gamma_{d}=\emptyset, H_{\Gamma_{d}}^{1}(\Omega)$ coincides with $H^{1}(\Omega)$. 
The variational formulation of problem (2.1) is given by

$$
\begin{aligned}
& \text { Find } p \in H_{\Gamma_{d}}^{1}(\Omega) \text { such that } \\
& a(p, q)=l(q), \quad \forall q \in H_{\Gamma_{d}}^{1}(\Omega) .
\end{aligned}
$$

$a(\cdot, \cdot)$ is a continuous sesquilinear form (linear in the first argument and conjugate linear in the second) defined on $H_{\Gamma_{d}}^{1}(\Omega) \times H_{\Gamma_{d}}^{1}(\Omega)$ by

$$
a(p, q)=\int_{\Omega}\left(\nabla p \cdot \nabla \bar{q}-k^{2} p \bar{q}\right)-\int_{\Gamma_{r}}(i \beta-\alpha) p \bar{q}
$$

where $\bar{q}$ is the complex conjugate of the complex-valued function $q . l$ is a continuous antilinear form (conjugate linear) defined on $H_{\Gamma_{d}}^{1}(\Omega)$ by

$$
l(q)=\int_{\Omega} f \bar{q}+\int_{\Gamma_{r}} g \bar{q} .
$$

Throughout the paper, we assume that the bounded coefficients $\alpha$ and $\beta$ satisfy

$$
|\alpha(\mathbf{x})| \leq C_{|\alpha|} \quad \forall \mathbf{x} \in \Gamma_{r}
$$

and

$$
0<C_{\beta,-} k \leq \beta(\mathbf{x}) \leq C_{\beta,+} k, \quad \forall \mathbf{x} \in \Gamma_{r},
$$

where the constants $C_{|\alpha|}, C_{\beta,-}$, and $C_{\beta,+}$ depend only on the domain $\Omega$. Examples of pairs for $(\alpha, \beta)$ include $(0, k)$ for the so-called Sommerfeld condition and, in two dimensions, the pair $(\zeta(\mathbf{x}) / 2, k)$ for the first order generalized Bayliss-GunzburgerTurkel condition $[1,2]$, where $\zeta$ is the curvature on $\Gamma_{r}$.

Finally, we recall the existence result:

Proposition 2.1. There exists a unique solution in $H_{\Gamma_{d}}^{1}(\Omega)$ of the variational problem (2.4).

This result is well known and, for a proof, we refer the reader to Ihlenburg [9] and the references therein.

\section{Stability}

In this section, we prove stability estimates for the unique solution $p$ of problem (2.4) for a class of bounded domains. For example, for any large wave number $k$, we will prove that $p$ satisfies

$$
k\|p\|_{0, \Omega}+|p|_{1, \Omega} \leq C\left(\|f\|_{0, \Omega}+\|g\|_{0, \Gamma_{r}}\right),
$$

where $C$ is a constant which does not depend on $k$.

3.1. State of the art. From the Banach-Nečas-Babuška theorem [6, Thm. 2.6], we can establish the continuous dependency of the unique solution with respect to the source terms. But to express the dependence of the upper bound on the wave number $k$, additional work is required. This dependence is important in the error analysis of a discretization method in order to define relations between the wave number and the discretization parameters.

To the best of our knowledge, on a bounded domain $\Omega$ sharp stability estimates with the explicit dependence on the wave number $k$ have only been shown 
- For problems in $\mathbb{R}$ : Douglas et al. [5] studied the problem with a Robin boundary condition $\left(\Gamma_{r}=\partial \Omega\right)$; Ihlenburg and Babuška [10] analyzed a problem with mixed boundary conditions $(\Omega=] 0,1\left[, \Gamma_{d}=\{0\}\right.$, and $\left.\Gamma_{r}=\{1\}\right)$.

- For problems with a Robin boundary condition $\left(\Gamma_{r}=\partial \Omega\right)$ : Melenk studied the two-dimensional problem in his thesis [11] with the assumption

$$
\mathbf{x} \cdot \boldsymbol{\nu}(\mathbf{x}) \geq \gamma>0, \quad \forall \mathbf{x} \in \partial \Omega
$$

Cummings and Feng [3] extended Melenk's proof to three-dimensional problems with the same assumption on the domain $\Omega$.

Actually, we can extend Melenk's proof to problems with mixed boundary conditions. We present this extension in the paper.

3.2. Technical results. We state two technical lemmas, which are used in the proof of estimate (3.1). We denote the Sobolev space of essentially bounded functions whose first derivatives are also essentially bounded by $W^{1, \infty}(\Omega)$.

Lemma 3.1. Let $\mathbf{m}$ be a field of real vectors in $W^{1, \infty}(\Omega)^{d}$. For all $q \in H^{1}(\Omega)$, we have

$$
\int_{\partial \Omega}|q|^{2} \mathbf{m} \cdot \boldsymbol{\nu}=\int_{\Omega} \operatorname{div}(\mathbf{m})|q|^{2}+2 \operatorname{Re} \int_{\Omega} q \mathbf{m} \cdot \nabla \bar{q},
$$

where $\operatorname{Re} z$ is the real part of the complex number $z$.

Proof. We have

$$
\int_{\Omega} \operatorname{div}\left(|q|^{2} \mathbf{m}\right)=\int_{\partial \Omega}|q|^{2} \mathbf{m} \cdot \boldsymbol{\nu} .
$$

We expand the divergence term to get

$$
\begin{aligned}
& \operatorname{div}\left(|q|^{2} \mathbf{m}\right)=|q|^{2} \operatorname{div}(\mathbf{m})+q \mathbf{m} \cdot \nabla \bar{q}+\bar{q} \mathbf{m} \cdot \nabla q, \\
& \operatorname{div}\left(|q|^{2} \mathbf{m}\right)=|q|^{2} \operatorname{div}(\mathbf{m})+2 \operatorname{Re}(q \mathbf{m} \cdot \nabla \bar{q}) .
\end{aligned}
$$

The following result is proved in Grisvard [7].

Lemma 3.2. Let $\mathbf{m}$ be a field of real vectors in $W^{1, \infty}(\Omega)^{d}$. For all $q \in H_{\Gamma_{d}}^{1}(\Omega) \cap$ $H^{3 / 2+\varepsilon}(\Omega)(\varepsilon>0)$, we have

$$
\begin{aligned}
& \int_{\partial \Omega \backslash \Gamma_{d}}|\nabla q|^{2} \mathbf{m} \cdot \boldsymbol{\nu}-\int_{\Gamma_{d}}\left|\partial_{\nu} q\right|^{2} \mathbf{m} \cdot \boldsymbol{\nu} \\
= & \int_{\Omega} \operatorname{div}(\mathbf{m})|\nabla q|^{2}-2 \operatorname{Re} \int_{\Omega} \nabla q \cdot(\nabla \bar{q} \cdot \nabla) \mathbf{m} \\
& \quad-2 \operatorname{Re} \int_{\Omega} \Delta q(\mathbf{m} \cdot \nabla \bar{q})+2 \operatorname{Re} \int_{\partial \Omega \backslash \Gamma_{d}} \partial_{\nu} q(\mathbf{m} \cdot \nabla \bar{q}) .
\end{aligned}
$$

Finally, we will make an extensive use of the classical inequality

$$
u v \leq \frac{\xi}{2} u^{2}+\frac{1}{2 \xi} v^{2}
$$

for positive numbers $u, v$, and $\xi \neq 0$. 
3.3. Geometric assumption. In order to prove stability estimates, we need to constrain the domain $\Omega$. We define the geometric assumption $(G A)$ as follows:

- The unique solution $p$ of problem (2.4) belongs to $H^{3 / 2+\varepsilon}(\Omega)$ with $\varepsilon>0$;

- There exists a point $\mathbf{x}_{0} \in \mathbb{R}^{d}$ and a constant $\gamma>0$ such that

$$
\begin{array}{ll}
\left(\mathbf{x}-\mathbf{x}_{0}\right) \cdot \boldsymbol{\nu}(\mathbf{x}) \leq 0 & \forall \mathbf{x} \in \Gamma_{d} \\
\left(\mathbf{x}-\mathbf{x}_{0}\right) \cdot \boldsymbol{\nu}(\mathbf{x})=0 & \forall \mathbf{x} \in \Gamma_{n} \\
\left(\mathbf{x}-\mathbf{x}_{0}\right) \cdot \boldsymbol{\nu}(\mathbf{x}) \geq \gamma & \forall \mathbf{x} \in \Gamma_{r}
\end{array}
$$

The regularity assumption on $p$ is actually a geometric constraint because of the regularity of the source terms $f$ and $g$ and the coefficients $\alpha$ and $\beta$. For example, with mixed boundary conditions, Grisvard [7, Lem. 5.1] states that the angles at the change of boundary conditions must be strictly convex.

The condition on $\Gamma_{r}$ is identical to the one of Melenk [11] and Cummings and Feng [3]. Grisvard [7, Eqn. (5.10)] used conditions similar to the ones on $\Gamma_{d}$ and on $\Gamma_{n}$ to deal with Dirichlet and Neumann boundary conditions. We remark that, in $\mathbb{R}^{2}$, $\Gamma_{n}$ is composed of straight segments.

When the boundary $\partial \Omega$ is connected and $\Gamma_{r}$ is different from $\partial \Omega$, the assumption $(G A)$ makes the product $\left(\mathbf{x}-\mathbf{x}_{0}\right) \cdot \boldsymbol{\nu}(\mathbf{x})$ discontinuous over $\partial \Omega$. Consequently, the domain $\Omega$ can not be smooth when the boundary $\partial \Omega$ is connected and $\Gamma_{r}$ is different from $\partial \Omega$.

We give now three examples of domains $\Omega$ satisfying the assumption $(G A)$.

- The first example models a scattering problem with a sound-soft scatterer. We set $\Omega=B(\mathbf{0}, 2) \backslash \overline{B(\mathbf{0}, 1)}$ (where $B(\mathbf{0}, 2)$ is the open ball of radius 2 centered at the origin). $\Gamma_{d}$ is the unit sphere and $\Gamma_{r}$ the sphere of radius 2. Here $\Gamma_{n}$ is empty and $\mathbf{x}_{0}$ coincides with the origin.

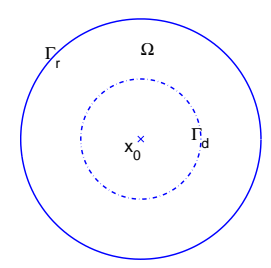

- For the second example, we set $\Omega$ to the L-shaped domain $] 0,1\left[{ }^{2} \backslash[1 / 2,1]^{2}\right.$. $\Gamma_{n}$ is $(\{0\} \times] 0,1[) \cup(] 0,1[\times\{0\})$ and $\Gamma_{r}$ the remaining part of the boundary. Here $\Gamma_{d}$ is empty and $\mathbf{x}_{0}$ coincides with the origin.

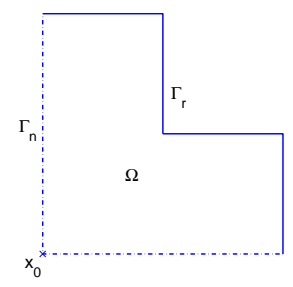

- The third example combines the three boundary conditions. 


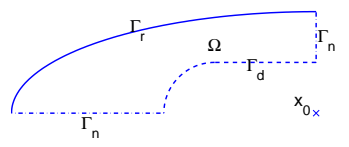

$\mathbf{x}_{0}$ coincides with the origin. $\Gamma_{n}$ is composed of two straight segments along the coordinate axes. $\Gamma_{r}$ is a quarter of an ellipse. $\Gamma_{d}$ is made of a segment and a quarter of a circle.

3.4. Stability estimates. In this section, we write our main stability results in two parts, one for large wave numbers and one for small wave numbers.

3.4.1. Case of a large wave number $k$. The proof is similar to the twodimensional proof of Melenk [11]. The difference lies in the treatment of mixed boundary conditions and of three-dimensional problems.

Proposition 3.3. Let $k_{0}$ be an arbitrary strictly positive number. If $\Omega$ satisfies the regularity assumption (GA), then there exists a constant $C$, which does not depend on $k$, such that for any $f \in L^{2}(\Omega), g \in L^{2}\left(\Gamma_{r}\right)$, and $k \geq k_{0}$, the unique solution $p$ of problem (2.4) satisfies

$$
k\|p\|_{0, \Omega}+|p|_{1, \Omega} \leq C\left(\|f\|_{0, \Omega}+\|g\|_{0, \Gamma_{r}}\right) .
$$

The constant $C$ depends on the domain $\Omega$, the constants $C_{|\alpha|}, C_{\beta,-}, C_{\beta,+}$, and $k_{0}$.

Proof. Throughout the proof, we use the notation $C$ to denote a generic positive constant depending only on the domain $\Omega$ and, when present, the subscript of $C$ refers to the origin of the constant.

First, we take the solution itself as a test function. The imaginary part of the resulting variational relation gives

$$
\begin{aligned}
\int_{\Gamma_{r}} \beta|p|^{2} & \leq\|f\|_{0, \Omega}\|p\|_{0, \Omega}+\|g\|_{0, \Gamma_{r}}\|p\|_{0, \Gamma_{r}}, \\
C_{\beta,-} k\|p\|_{0, \Gamma_{r}}^{2} & \leq \frac{1}{2 \xi_{1} k}\|f\|_{0, \Omega}^{2}+\frac{\xi_{1} k}{2}\|p\|_{0, \Omega}^{2}+\frac{1}{2 \xi_{2}}\|g\|_{0, \Gamma_{r}}^{2}+\frac{\xi_{2}}{2}\|p\|_{0, \Gamma_{r}}^{2}, \\
k^{2}\|p\|_{0, \Gamma_{r}}^{2} & \leq \frac{1}{C_{\beta,-}}\left(\frac{1}{2 \xi_{1}}\|f\|_{0, \Omega}^{2}+\frac{\xi_{1} k^{2}}{2}\|p\|_{0, \Omega}^{2}+\frac{k}{2 \xi_{2}}\|g\|_{0, \Gamma_{r}}^{2}+\frac{\xi_{2} k}{2}\|p\|_{0, \Gamma_{r}}^{2}\right) .
\end{aligned}
$$

Now we set $\xi_{2}$ to $k C_{\beta,-}$ to get

$$
\frac{k^{2}}{2}\|p\|_{0, \Gamma_{r}}^{2} \leq \frac{1}{C_{\beta,-}}\left(\frac{1}{2 \xi_{1}}\|f\|_{0, \Omega}^{2}+\frac{\xi_{1} k^{2}}{2}\|p\|_{0, \Omega}^{2}\right)+\frac{1}{2 C_{\beta,-}^{2}}\|g\|_{0, \Gamma_{r}}^{2} .
$$

Thus we obtain, for any $\epsilon>0$,

$$
k^{2}\|p\|_{0, \Gamma_{r}}^{2} \leq \frac{C}{\epsilon}\|f\|_{0, \Omega}^{2}+\epsilon k^{2}\|p\|_{0, \Omega}^{2}+C\|g\|_{0, \Gamma_{r}}^{2} .
$$

The real part of the same variational relation gives

$$
|p|_{1, \Omega}^{2}+\int_{\Gamma_{r}} \alpha|p|^{2} \leq k^{2}\|p\|_{0, \Omega}^{2}+\|f\|_{0, \Omega}\|p\|_{0, \Omega}+\|g\|_{0, \Gamma_{r}}\|p\|_{0, \Gamma_{r}} .
$$


Using the inequality (3.5), we write

$|p|_{1, \Omega}^{2} \leq\left(k^{2}+\frac{\xi_{3} k^{2}}{2}\right)\|p\|_{0, \Omega}^{2}+\frac{1}{2 \xi_{3} k^{2}}\|f\|_{0, \Omega}^{2}+\frac{1}{4 k^{2}}\|g\|_{0, \Gamma_{r}}^{2}+k^{2}\|p\|_{0, \Gamma_{r}}^{2}+C_{|\alpha|}\|p\|_{0, \Gamma_{r}}^{2}$.

We rewrite expression (3.8) as follows

$\left(k^{2}+C_{|\alpha|}\right)\|p\|_{0, \Gamma_{r}}^{2} \leq \frac{C}{\epsilon}\left(1+\frac{C_{|\alpha|}}{k^{2}}\right)\|f\|_{0, \Omega}^{2}+\epsilon\left(k^{2}+C_{|\alpha|}\right)\|p\|_{0, \Omega}^{2}+C\left(1+\frac{C_{|\alpha|}}{k^{2}}\right)\|g\|_{0, \Gamma_{r}}^{2}$.

Inserting this expression with $\epsilon=\xi_{4} k^{2} /\left(k^{2}+C_{|\alpha|}\right)$, we obtain

$$
\begin{aligned}
|p|_{1, \Omega}^{2} \leq k^{2}\left(1+\xi_{4}+\frac{\xi_{3}}{2}\right)\|p\|_{0, \Omega}^{2} & +\left(\frac{1}{2 \xi_{3} k^{2}}+\frac{C_{(3.8)}}{\xi_{4}} \frac{\left(k^{2}+C_{|\alpha|}\right)^{2}}{k^{4}}\right)\|f\|_{0, \Omega}^{2} \\
+ & C\left(1+\frac{1}{k^{2}}\right)\|g\|_{0, \Gamma_{r}}^{2} .
\end{aligned}
$$

In particular, when $\xi_{4}=\xi_{3} / 2=1 / 2$, we have

$$
|p|_{1, \Omega}^{2} \leq 2 k^{2}\|p\|_{0, \Omega}^{2}+C\left(1+\frac{1}{k^{2}}+\frac{1}{k^{4}}\right)\|f\|_{0, \Omega}^{2}+C\left(1+\frac{1}{k^{2}}\right)\|g\|_{0, \Gamma_{r}}^{2} .
$$

We now use expression (3.4) where we replace $-\Delta p$ with $f+k^{2} p$ in $\Omega, \partial_{\nu} p$ with 0 on $\Gamma_{n}$, and $\partial_{\nu} p$ with $(i \beta-\alpha) p+g$ on $\Gamma_{r}$.

$$
\begin{aligned}
& \int_{\partial \Omega \backslash \Gamma_{d}}|\nabla p|^{2} \mathbf{m} \cdot \boldsymbol{\nu}-\int_{\Gamma_{d}}\left|\partial_{\nu} p\right|^{2} \mathbf{m} \cdot \boldsymbol{\nu} \\
= & \int_{\Omega} \operatorname{div}(\mathbf{m})|\nabla p|^{2}-2 \operatorname{Re} \int_{\Omega} \nabla p \cdot(\nabla \bar{p} \cdot \nabla) \mathbf{m} \\
& +2 \operatorname{Re} \int_{\Omega}\left(f+k^{2} p\right)(\mathbf{m} \cdot \nabla \bar{p})+2 \operatorname{Re} \int_{\Gamma_{r}}((i \beta-\alpha) p+g)(\mathbf{m} \cdot \nabla \bar{p}) .
\end{aligned}
$$

We replace $\operatorname{Re} \int_{\Omega} p(\mathbf{m} \cdot \nabla \bar{p})$ with the relationship (3.3):

$$
\begin{aligned}
& \int_{\partial \Omega \backslash \Gamma_{d}}|\nabla p|^{2} \mathbf{m} \cdot \boldsymbol{\nu}-\int_{\Gamma_{d}}\left|\partial_{\nu} p\right|^{2} \mathbf{m} \cdot \boldsymbol{\nu} \\
= & \int_{\Omega} \operatorname{div}(\mathbf{m})|\nabla p|^{2}-2 \operatorname{Re} \int_{\Omega} \nabla p \cdot(\nabla \bar{p} \cdot \nabla) \mathbf{m} \\
& +2 \operatorname{Re} \int_{\Omega} f(\mathbf{m} \cdot \nabla \bar{p})+k^{2} \int_{\partial \Omega}|p|^{2} \mathbf{m} \cdot \boldsymbol{\nu} \\
& -k^{2} \int_{\Omega} \operatorname{div}(\mathbf{m})|p|^{2}+2 \operatorname{Re} \int_{\Gamma_{r}}((i \beta-\alpha) p+g)(\mathbf{m} \cdot \nabla \bar{p}) .
\end{aligned}
$$

Inserting the Dirichlet boundary condition on $\Gamma_{d}$, we now obtain

$$
\begin{aligned}
& -\int_{\Gamma_{d}}\left|\partial_{\nu} p\right|^{2} \mathbf{m} \cdot \boldsymbol{\nu}+\int_{\Gamma_{n}}|\nabla p|^{2} \mathbf{m} \cdot \boldsymbol{\nu}+\int_{\Gamma_{r}}|\nabla p|^{2} \mathbf{m} \cdot \boldsymbol{\nu}+k^{2} \int_{\Omega} \operatorname{div}(\mathbf{m})|p|^{2} \\
= & \int_{\Omega} \operatorname{div}(\mathbf{m})|\nabla p|^{2}-2 \operatorname{Re} \int_{\Omega} \nabla p \cdot(\nabla \bar{p} \cdot \nabla) \mathbf{m}+2 \operatorname{Re} \int_{\Omega} f(\mathbf{m} \cdot \nabla \bar{p}) \\
& +k^{2} \int_{\Gamma_{n}}|p|^{2} \mathbf{m} \cdot \boldsymbol{\nu}+k^{2} \int_{\Gamma_{r}}|p|^{2} \mathbf{m} \cdot \boldsymbol{\nu}+2 \operatorname{Re} \int_{\Gamma_{r}}((i \beta-\alpha) p+g)(\mathbf{m} \cdot \nabla \bar{p}) .
\end{aligned}
$$


We replace the vector field $\mathbf{m}$ with $\mathbf{x}-\mathbf{x}_{0}$ and we have

$$
\begin{array}{rll}
\operatorname{div}\left(\mathbf{x}-\mathbf{x}_{0}\right) & =d & \text { in } \Omega, \\
\nabla p \cdot(\nabla \bar{p} \cdot \nabla)\left(\mathbf{x}-\mathbf{x}_{0}\right) & =|\nabla p|^{2} & \text { in } \Omega, \\
\left(\mathbf{x}-\mathbf{x}_{0}\right) \cdot \boldsymbol{\nu}(\mathbf{x}) & \leq 0 & \text { on } \Gamma_{d}, \\
\left(\mathbf{x}-\mathbf{x}_{0}\right) \cdot \boldsymbol{\nu}(\mathbf{x}) & =0 & \text { on } \Gamma_{n}, \\
\left(\mathbf{x}-\mathbf{x}_{0}\right) \cdot \boldsymbol{\nu}(\mathbf{x}) & \geq \gamma & \text { on } \Gamma_{r},
\end{array}
$$

where $\gamma$ is a strictly positive constant. As $\Omega$ is a bounded domain, we get

$$
\begin{aligned}
d k^{2}\|p\|_{0, \Omega}^{2}+\gamma\|\nabla p\|_{0, \Gamma_{r}}^{2} \leq & (d-2)|p|_{1, \Omega}^{2}+C\left(\|f\|_{0, \Omega}|p|_{1, \Omega}+\|g\|_{0, \Gamma_{r}}\|\nabla p\|_{0, \Gamma_{r}}\right) \\
& +C\left(k^{2}\|p\|_{0, \Gamma_{r}}^{2}+\sup _{\Gamma_{r}}|i \beta-\alpha|\|p\|_{0, \Gamma_{r}}\|\nabla p\|_{0, \Gamma_{r}}\right) .
\end{aligned}
$$

To eliminate $\|\nabla p\|_{0, \Gamma_{r}}^{2}$ in the left-hand side of (3.13), we use

$$
\begin{aligned}
\sup _{\Gamma_{r}}|i \beta-\alpha|\|p\|_{0, \Gamma_{r}}\|\nabla p\|_{0, \Gamma_{r}} & \leq \frac{k^{2}}{2 \xi_{5}}\|p\|_{0, \Gamma_{r}}^{2}+\frac{\xi_{5}\left(C_{\beta,+}^{2} k^{2}+C_{|\alpha|}^{2}\right)}{2 k^{2}}\|\nabla p\|_{0, \Gamma_{r}}^{2} \\
\|g\|_{0, \Gamma_{r}}\|\nabla p\|_{0, \Gamma_{r}} & \leq \frac{1}{2 \xi_{6}}\|g\|_{0, \Gamma_{r}}^{2}+\frac{\xi_{6}}{2}\|\nabla p\|_{0, \Gamma_{r}}^{2}
\end{aligned}
$$

with $\xi_{5}$ and $\xi_{6}$ satisfying

$$
\frac{\gamma}{2}=C_{(3.13)} \frac{\xi_{5}\left(C_{\beta,+}^{2} k^{2}+C_{|\alpha|}^{2}\right)}{2 k^{2}}=C_{(3.13)} \frac{\xi_{6}}{2} .
$$

We note that $\xi_{5}$ satisfies also

$$
\frac{k^{2}}{2 \xi_{5}}=\frac{C_{(3.13)}}{2 \gamma}\left(C_{\beta,+}^{2} k^{2}+C_{|\alpha|}^{2}\right) \leq C\left(k^{2}+1\right) .
$$

We now obtain

$$
d k^{2}\|p\|_{0, \Omega}^{2} \leq C\left[\left(k^{2}+1\right)\|p\|_{0, \Gamma_{r}}^{2}+\|f\|_{0, \Omega}|p|_{1, \Omega}+\|g\|_{0, \Gamma_{r}}^{2}\right]+(d-2)|p|_{1, \Omega}^{2} .
$$

We rewrite (3.8) with $\epsilon=k^{2} \xi_{7} /\left(k^{2}+1\right)$ :

$$
\left(k^{2}+1\right)\|p\|_{0, \Gamma_{r}}^{2} \leq \frac{C_{(3.8)}}{\xi_{7}} \frac{\left(k^{2}+1\right)^{2}}{k^{4}}\|f\|_{0, \Omega}^{2}+\xi_{7} k^{2}\|p\|_{0, \Omega}^{2}+C_{(3.8)}\left(1+\frac{1}{k^{2}}\right)\|g\|_{0, \Gamma_{r}}^{2} .
$$

Inserting this relation into (3.15), we have

$$
\begin{aligned}
k^{2}\left(d-\xi_{7} C_{(3.15)}\right)\|p\|_{0, \Omega}^{2} \leq C\left(\|f\|_{0, \Omega}|p|_{1, \Omega}+\|g\|_{0, \Gamma_{r}}^{2}\right)+(d-2)|p|_{1, \Omega}^{2} & \\
& +\frac{C}{\xi_{7}} \frac{\left(k^{2}+1\right)^{2}}{k^{4}}\|f\|_{0, \Omega}^{2}+C\left(1+\frac{1}{k^{2}}\right)\|g\|_{0, \Gamma_{r}}^{2}
\end{aligned}
$$

that we combine with expression (3.10) and $\xi_{4}=\xi_{3} / 2=\xi_{8}$ to obtain

$$
\begin{array}{r}
k^{2}\left[2-\xi_{7} C_{(3.15)}-2(d-2) \xi_{8}\right]\|p\|_{0, \Omega}^{2} \leq C\|f\|_{0, \Omega}|p|_{1, \Omega}+C\left(1+\frac{1}{k^{2}}\right)\|g\|_{0, \Gamma_{r}}^{2} \\
+C\left(\frac{1}{\xi_{7}} \frac{\left(k^{2}+1\right)^{2}}{k^{4}}+\frac{1}{\xi_{8}}\left(1+\frac{1}{k^{2}}+\frac{1}{k^{4}}\right)\right)\|f\|_{0, \Omega}^{2} .
\end{array}
$$


Next, we deal with the term $\|f\|_{0, \Omega}|p|_{1, \Omega}$ as follows:

$$
\begin{aligned}
\|f\|_{0, \Omega}|p|_{1, \Omega} \leq & \frac{1}{2 \xi_{9}}\|f\|_{0, \Omega}^{2}+\frac{\xi_{9}}{2}|p|_{1, \Omega}^{2} \\
\|f\|_{0, \Omega}|p|_{1, \Omega} \leq & \xi_{9} k^{2}\|p\|_{0, \Omega}^{2}+\left[\frac{1}{2 \xi_{9}}+C \xi_{9}\left(1+\frac{1}{k^{2}}+\frac{1}{k^{4}}\right)\right]\|f\|_{0, \Omega}^{2} \\
& +C \xi_{9}\left(1+\frac{1}{k^{2}}\right)\|g\|_{0, \Gamma_{r}}^{2}
\end{aligned}
$$

where we used (3.11). Inserting the latest bound in (3.17) results in

$$
\begin{array}{r}
k^{2}\left[2-\xi_{7} C_{(3.15)}-2(d-2) \xi_{8}-C_{(3.17)} \xi_{9}\right]\|p\|_{0, \Omega}^{2} \leq C\left(1+\xi_{9}\right)\left(1+\frac{1}{k^{2}}\right)\|g\|_{0, \Gamma_{r}}^{2} \\
+C\left[\frac{1}{\xi_{7}} \frac{\left(k^{2}+1\right)^{2}}{k^{4}}+\frac{1}{\xi_{9}}+\left(\frac{1}{\xi_{8}}+\xi_{9}\right)\left(1+\frac{1}{k^{2}}+\frac{1}{k^{4}}\right)\right]\|f\|_{0, \Omega}^{2} .
\end{array}
$$

By selecting $\xi_{7}, \xi_{8}$, and $\xi_{9}$ such that

$$
2-\xi_{7} C_{(3.15)}-2(d-2) \xi_{8}-C_{(3.17)} \xi_{9}=1,
$$

we obtain

$$
k^{2}\|p\|_{0, \Omega}^{2} \leq C\left(1+\frac{1}{k^{2}}+\frac{1}{k^{4}}\right)\left(\|f\|_{0, \Omega}^{2}+\|g\|_{0, \Gamma_{r}}^{2}\right) .
$$

Furthermore, when using expression (3.11), we have

$$
|p|_{1, \Omega}^{2} \leq C\left(1+\frac{1}{k^{2}}+\frac{1}{k^{4}}\right)\left(\|f\|_{0, \Omega}^{2}+\|g\|_{0, \Gamma_{r}}^{2}\right) .
$$

These last two bounds give (3.7), provided that the wave number $k$ stays away from 0 .

When only a Robin boundary condition is specified (i.e. $\Gamma_{d}=\Gamma_{n}=\emptyset$ ) and the coefficients $(\alpha, \beta)$ are equal to $(0, k)$, we recover the optimal results of Melenk [11] and of Cummings and Feng [3].

The result (3.7) is new for two-dimensional and three-dimensional domains with mixed boundary conditions. The bounds are similar to the optimal one-dimensional result of Ihlenburg and Babuška [10] and, consequently, are sharp with respect to the wave number $k$.

3.4.2. Case of a small wave number $k$. To study the case for small values of $k$, we consider the following boundary value problem:

$$
\left\{\begin{aligned}
-\Delta \phi & =f & & \text { in } \Omega, \\
\phi & =0 & & \text { on } \Gamma_{d}, \\
\partial_{\nu} \phi & =0 & & \text { on } \Gamma_{n} \\
\partial_{\nu} \phi & =-\alpha \phi+g & & \text { on } \Gamma_{r} .
\end{aligned}\right.
$$

Formally, this problem is the limit when $k$ is zero for the Helmholtz problem (2.1). The variational formulation of problem (3.21) is given by

Find $\phi \in H_{\Gamma_{d}}^{1}(\Omega)$ such that

$$
a_{0}(\phi, \psi)=l(\psi), \quad \forall \psi \in H_{\Gamma_{d}}^{1}(\Omega) .
$$


$a_{0}(\cdot, \cdot)$ is a continuous sesquilinear form (linear in the first argument and conjugate linear in the second) defined on $H_{\Gamma_{d}}^{1}(\Omega) \times H_{\Gamma_{d}}^{1}(\Omega)$ by

$$
a_{0}(\phi, \psi)=\int_{\Omega} \nabla \phi \cdot \nabla \bar{\psi}+\int_{\Gamma_{r}} \alpha \phi \bar{\psi} .
$$

$l$ is the continuous antilinear form defined by (2.6).

The bilinear form $a_{0}$ satisfies a Gärding inequality. Indeed, we have

$$
\left.\left|\int_{\Gamma_{r}} \alpha\right| \psi\right|^{2} \mid \leq C_{|\alpha|}\|\psi\|_{0, \Gamma_{r}}^{2} \leq C\|\psi\|_{1, \Omega}\|\psi\|_{0, \Omega} \leq C \varepsilon\|\psi\|_{1, \Omega}^{2}+\frac{C}{\varepsilon}\|\psi\|_{0, \Omega}^{2}
$$

and

$$
a_{0}(\psi, \psi)=\int_{\Omega}|\nabla \psi|^{2}+\int_{\Gamma_{r}} \alpha|\psi|^{2} \geq(1-C \varepsilon)|\psi|_{1, \Omega}^{2}-C\left(\varepsilon+\frac{1}{\varepsilon}\right)\|\psi\|_{0, \Omega}^{2} .
$$

Consequently, the Fredholm alternative applies to the form $a_{0}$. To prove stability estimates for small wave numbers, we distinguish whether problem (3.22) has a unique solution or not.

Proposition 3.4. When problem (3.22) is well-posed, there exists a positive number $k_{0}$ and a constant $C$, which does not depend on $k$, such that for any $f \in L^{2}(\Omega)$, $g \in L^{2}\left(\Gamma_{r}\right)$, and $k \leq k_{0}$, the unique solution $p$ of problem (2.4) satisfies

$$
\|p\|_{1, \Omega} \leq C\left(\|f\|_{0, \Omega}+\|g\|_{0, \Gamma_{r}}\right) .
$$

The constant $C$ depends only on the domain $\Omega$ and the constants $C_{|\alpha|}, C_{\beta,-}, C_{\beta,+}$, and $k_{0}$.

Proof. Problem (3.22) is well-posed, thus we can apply the Banach-Nečas-Babuška theorem [6, Thm. 2.6]. Thus there exists a constant $C$ such that

$$
\|\phi\|_{1, \Omega} \leq C\left(\|f\|_{0, \Omega}+\|g\|_{0, \Gamma_{r}}\right) .
$$

The solution $p$ of problem (2.4) satisfies problem (3.21) with right hand sides of $f+k^{2} p$ and $g+i \beta p$. Consequently, we have

$$
\|p\|_{1, \Omega} \leq C\left(\left\|f+k^{2} p\right\|_{0, \Omega}+\|g+i \beta p\|_{0, \Gamma_{r}}\right) .
$$

Also, we have

$$
\|p\|_{0, \Gamma_{r}} \leq C\|p\|_{1, \Omega}
$$

Thus we obtain

$$
\left(1-C_{(3.25)} k^{2}-C_{(3.26)} C_{\beta,+} k\right)\|p\|_{1, \Omega} \leq C\left(\|f\|_{0, \Omega}+\|g\|_{0, \Gamma_{r}}\right) .
$$

We conclude that, for $k$ sufficiently small, we have

$$
\|p\|_{1, \Omega} \leq C\left(\|f\|_{0, \Omega}+\|g\|_{0, \Gamma_{r}}\right) .
$$

We remark that the result (3.24) does not require the geometric assumption $(G A)$. Examples of a well-posed problem (3.22) include the case where $\alpha>0$ and the case where $\alpha \geq 0$ and $\Gamma_{d} \neq \emptyset$. 
When problem (3.21) is not well-posed, the $L^{2}$-norm of $p$ should not remain bounded as the wave number $k$ goes to zero. For general cases, we were not able to prove this result. However, for the particular case where $\alpha$ is zero and $\Gamma_{d}$ is empty, we can give sharp upper bounds. This result does not require the geometric assumption $(G A)$.

Proposition 3.5. When $\alpha$ is zero and $\Gamma_{d}$ is empty, there exists a positive number $k_{0}$ and a constant $C$, which does not depend on $k$, such that for any $f \in L^{2}(\Omega)$, $g \in L^{2}\left(\Gamma_{r}\right)$, and $k \leq k_{0}$, the unique solution $p$ of problem (2.4) satisfies

$$
k\|p\|_{0, \Omega}+|p|_{1, \Omega} \leq C\left(\|f\|_{0, \Omega}+\|g\|_{0, \Gamma_{r}}\right) .
$$

The constant $C$ depends only on the domain $\Omega$ and the constants $C_{|\alpha|}, C_{\beta,-}, C_{\beta,+}$, and $k_{0}$.

Proof. We introduce the average $\underline{p}$,

$$
\underline{p}=\frac{1}{\operatorname{meas}(\Omega)} \int_{\Omega} p .
$$

We use the function $p-\underline{p}$ inside the sesquilinear form $a$ :

$$
\begin{gathered}
a(p-\underline{p}, p-\underline{p})=a(p, p-\underline{p})-a(\underline{p}, p-\underline{p}) \\
a(p-\underline{p}, p-\underline{p})=\int_{\Omega} f(\overline{p-\underline{p}})+\int_{\Gamma_{r}} g(\overline{p-\underline{p}})+k^{2} \int_{\Omega} \underline{p}(\overline{p-\underline{p}})+\int_{\Gamma_{r}} i \beta \underline{p}(\overline{p-\underline{p}}) .
\end{gathered}
$$

Noting that

$$
\int_{\Omega} \underline{p}(\overline{p-\underline{p}})=0
$$

the real part of the variational relation (3.28) gives

$$
\begin{aligned}
|p|_{1, \Omega}^{2}-k^{2}\|p-\underline{p}\|_{0, \Omega}^{2} \leq\|f\|_{0, \Omega}\|p-\underline{p}\|_{0, \Omega} & +\|g\|_{0, \Gamma_{r}}\|p-\underline{p}\|_{0, \Gamma_{r}} \\
& +C_{\beta,+} k|\underline{p}|\|p-\underline{p}\|_{0, \Gamma_{r}},
\end{aligned}
$$

where we used the upper bound on $\beta$. We have also

$$
\|p-\underline{p}\|_{0, \Omega} \leq C|p|_{1, \Omega} \text { and }\|p-\underline{p}\|_{0, \Gamma_{r}} \leq C|p|_{1, \Omega} .
$$

For small $k$, we combine (3.29) and (3.30):

$$
\begin{aligned}
& |p|_{1, \Omega}^{2} \leq C\left(\|f\|_{0, \Omega}|p|_{1, \Omega}+\|g\|_{0, \Gamma_{r}}|p|_{1, \Omega}+k|\underline{p}||p|_{1, \Omega}\right) \\
& |p|_{1, \Omega}^{2} \leq C\left(\|f\|_{0, \Omega}+\|g\|_{0, \Gamma_{r}}\right)|p|_{1, \Omega}+\frac{C^{2} k^{2}}{2}|\underline{p}|^{2}+\frac{1}{2}|p|_{1, \Omega}^{2} .
\end{aligned}
$$

Finally, we get

$$
|p|_{1, \Omega}^{2} \leq C\left(\|f\|_{0, \Omega}+\|g\|_{0, \Gamma_{r}}\right)|p|_{1, \Omega}+C k^{2}|\underline{p}|^{2},
$$

where $C$ is a generic constant depending only on $\Omega$. So we need now to estimate $k^{2}|\underline{p}|^{2}$. 
As $\sqrt{|p|_{1, \Omega}^{2}+\|p\|_{0, \Gamma_{r}}^{2}}$ is a norm on $H^{1}(\Omega)$, we write

$$
|\underline{p}|^{2} \leq C\left(\|p\|_{0, \Gamma_{r}}^{2}+|p|_{1, \Omega}^{2}\right) .
$$

Then, in order to bound $\|p\|_{0, \Gamma_{r}}$, we start by using $p-\underline{p}$ as a test function. The imaginary part of the variational relation gives

$$
\int_{\Gamma_{r}} \beta|p|^{2} \leq C\left(\|f\|_{0, \Omega}+\|g\|_{0, \Gamma_{r}}\right)|p|_{1, \Omega}+\left|\operatorname{Im}\left[\int_{\Gamma_{r}} i \beta p \underline{\bar{p}}\right]\right| .
$$

Similarly, when $\underline{p}$ is the test function, the imaginary part results in

$$
\left|\operatorname{Im}\left[\int_{\Gamma_{r}} i \beta p \bar{p}\right]\right| \leq\left(\|f\|_{0, \Omega}+\|g\|_{0, \Gamma_{r}}\right)|\underline{p}| .
$$

Thus we get

$$
k\|p\|_{0, \Gamma_{r}}^{2} \leq C\left(\|f\|_{0, \Omega}+\|g\|_{0, \Gamma_{r}}\right)\left(|p|_{1, \Omega}+|\underline{p}|\right) .
$$

With this upper bound, relation (3.32) now becomes

$$
k^{2}|\underline{p}|^{2} \leq C k^{2}|p|_{1, \Omega}^{2}+C k\left(\|f\|_{0, \Omega}+\|g\|_{0, \Gamma_{r}}\right)\left(|p|_{1, \Omega}+|\underline{p}|\right)
$$

and

$$
\begin{aligned}
k^{2}|\underline{p}|^{2} \leq C_{(3.35)} k^{2}|p|_{1, \Omega}^{2}+\frac{C_{(3.35)}}{2}\left(\|f\|_{0, \Omega}+\|g\|_{0, \Gamma_{r}}\right)^{2}+C_{(3.35)} \frac{k^{2}}{2}|p|_{1, \Omega}^{2} \\
\quad+\frac{k^{2}}{2}|\underline{p}|^{2}+\frac{C_{(3.35)}^{2}}{2}\left(\|f\|_{0, \Omega}+\|g\|_{0, \Gamma_{r}}\right)^{2} \\
k^{2}|\underline{p}|^{2} \leq C k^{2}|p|_{1, \Omega}^{2}+C\left(\|f\|_{0, \Omega}+\|g\|_{0, \Gamma_{r}}\right)^{2} .
\end{aligned}
$$

We insert this result into (3.31) so that we have

$$
\begin{aligned}
& |p|_{1, \Omega}^{2} \leq C\left(\|f\|_{0, \Omega}+\|g\|_{0, \Gamma_{r}}\right)|p|_{1, \Omega}+C k^{2}|p|_{1, \Omega}^{2}+C\left(\|f\|_{0, \Omega}+\|g\|_{0, \Gamma_{r}}\right)^{2}, \\
& |p|_{1, \Omega}^{2} \leq C\left(\|f\|_{0, \Omega}+\|g\|_{0, \Gamma_{r}}\right)^{2}+\frac{1}{2}|p|_{1, \Omega}^{2}+C k^{2}|p|_{1, \Omega}^{2} .
\end{aligned}
$$

For small values of $k$, we now obtain

$$
|p|_{1, \Omega}^{2} \leq C\left(\|f\|_{0, \Omega}+\|g\|_{0, \Gamma_{r}}\right)^{2} .
$$

Finally, in order to bound $k^{2}\|p\|_{0, \Omega}^{2}$, we write

$$
k^{2}\|p\|_{0, \Omega}^{2} \leq C k^{2}\left(\|p-\underline{p}\|_{0, \Omega}^{2}+|\underline{p}|^{2}\right) \leq C k^{2}\left(|p|_{1, \Omega}^{2}+|\underline{p}|^{2}\right)
$$

and we use (3.36) and (3.37) to bound $k^{2}\|p\|_{0, \Omega}^{2}$ for small values of the wave number $k$.

When only a Robin boundary condition is specified (i.e. $\Gamma_{d}=\Gamma_{n}=\emptyset$ ) and the coefficients $(\alpha, \beta)$ are equal to $(0, k)$, Prop. 3.5 states that the $L^{2}$-norm of the solution gradient remains bounded when the wave number approaches zero. This result is new for three-dimensional domains as this boundedness was not proved by Cummings and Feng [3]. 
3.4.3. Case of a smooth solution $p$. Finally, we state a stability estimate when $p$ belongs to $H^{2}(\Omega)$.

Proposition 3.6. If the source term $g$ belongs to $H^{1 / 2}\left(\Gamma_{r}\right)$ and the solution $p$ belongs to $H^{2}(\Omega)$, then we have

$$
|p|_{2, \Omega} \leq C\left(\|f\|_{0, \Omega}+\|g\|_{1 / 2, \Gamma_{r}}\right)+C(1+k)\left(k\|p\|_{0, \Omega}+|p|_{1, \Omega}\right)
$$

where $C$ does not depend on $k$.

Proof. The application

$$
\mathcal{L}:\left\{\begin{aligned}
H_{\Gamma_{d}}^{1}(\Omega) \cap H^{2}(\Omega) & \rightarrow L^{2}(\Omega) \times H^{1 / 2}\left(\Gamma_{n}\right) \times H^{1 / 2}\left(\Gamma_{r}\right) \\
\psi & \mapsto\left(-\Delta \psi, \partial_{\nu} \psi, \partial_{\nu} \psi+\alpha \psi\right)
\end{aligned}\right.
$$

is continuous and surjective. We apply a corollary of the open mapping theorem, described in Dautray and Lions [4, Corollary 2 on page 279], to define a continuous bijective mapping from the quotient-space

$$
\left(H_{\Gamma_{d}}^{1}(\Omega) \cap H^{2}(\Omega)\right) / \operatorname{Ker} \mathcal{L}
$$

into the space

$$
L^{2}(\Omega) \times H^{1 / 2}\left(\Gamma_{n}\right) \times H^{1 / 2}\left(\Gamma_{r}\right)
$$

The mapping

$$
\psi \mapsto \sqrt{|\psi|_{1, \Omega}^{2}+|\psi|_{2, \Omega}^{2}+\int_{\Gamma_{r}}|\psi|^{2}}
$$

defines a norm on this quotient-space. Thus we have

$$
\sqrt{|\psi|_{1, \Omega}^{2}+|\psi|_{2, \Omega}^{2}+\int_{\Gamma_{r}}|\psi|^{2}} \leq C\left(\|-\Delta \psi\|_{0, \Omega}+\left\|\partial_{\nu} \psi\right\|_{1 / 2, \Gamma_{n}}+\left\|\partial_{\nu} \psi+\alpha \psi\right\|_{1 / 2, \Gamma_{r}}\right)
$$

where $C$ is a constant depending only on the domain $\Omega$ and the coefficient $\alpha$.

We now obtain, for the solution $p$,

$$
\begin{aligned}
& |p|_{2, \Omega} \leq C\left(\|-\Delta p\|_{0, \Omega}+\left\|\partial_{\nu} p\right\|_{1 / 2, \Gamma_{n}}+\left\|\partial_{\nu} p+\alpha p\right\|_{1 / 2, \Gamma_{r}}\right) \\
& |p|_{2, \Omega} \leq C\left(\left\|f+k^{2} p\right\|_{0, \Omega}+\|i \beta p+g\|_{1 / 2, \Gamma_{r}}\right) \\
& |p|_{2, \Omega} \leq C\left(\|f\|_{0, \Omega}+k^{2}\|p\|_{0, \Omega}+k\|p\|_{1 / 2, \Gamma_{r}}+\|g\|_{1 / 2, \Gamma_{r}}\right) \\
& |p|_{2, \Omega} \leq C\left[\|f\|_{0, \Omega}+k^{2}\|p\|_{0, \Omega}+k\left(\|p\|_{0, \Omega}+|p|_{1, \Omega}\right)+\|g\|_{1 / 2, \Gamma_{r}}\right] \\
& |p|_{2, \Omega} \leq C\left(\|f\|_{0, \Omega}+\|g\|_{1 / 2, \Gamma_{r}}\right)+C\left(k+k^{2}\right)\|p\|_{0, \Omega}+C k|p|_{1, \Omega} .
\end{aligned}
$$

Prop. 3.6 does not require the geometric assumption $(G A)$ and is valid for any wave number $k$. When the solution $p$ satisfies the stability estimate

$$
k\|p\|_{0, \Omega}+|p|_{1, \Omega} \leq C\left(\|f\|_{0, \Omega}+\|g\|_{0, \Gamma_{r}}\right),
$$

we obtain

$$
|p|_{2, \Omega} \leq C(1+k)\left(\|f\|_{0, \Omega}+\|g\|_{1 / 2, \Gamma_{r}}\right) .
$$


Acknowledgments. The author thanks Prof. R. Djellouli (CSU Northridge) and the anonymous referees for comments that led to improvements of the manuscript.

\section{REFERENCES}

[1] X. Antoine, H. Barucq and A. Bendali, Bayliss-Turkel like radiation conditions on surfaces of arbitrary shape, J. Math. Anal. Appl., 229, 184-211, 1999.

[2] A. Bayliss, M. Gunzburger and E. Turkel, Boundary conditions for the numerical solution of elliptic equations in exterior regions, SIAM J. Appl. Math., 42, 430-451, 1982.

[3] P. Cummings and X. Feng, Sharp regularity coefficient estimates for complex-valued acoustic and elastic Helmholtz equations, Math. Models Methods. Appl. Sci., 16(1), 139-160, 2006.

[4] R. Dautray and J. Lions, Mathematical Analysis and Numerical Methods for Science and Technology, Springer, 2, 1988.

[5] J. Douglas, J. Santos, D. Sheen and L. Bennethum, Frequency domain treatment of onedimensional scalar waves, Math. Models Methods. Appl. Sci., 3(2), 171-194, 1993.

[6] A. Ern and J.L. Guermond, Theory and Practice of Finite Elements, Appl. Math. Sci., Springer, $159,2004$.

[7] P. Grisvard, Contrôlabilité exacte des solutions de l'équation des ondes enprésence de singularités, J. Math. Pures et Appl., 68, 215-259, 1989.

[8] U. Hetmaniuk, Fictitious domain decomposition methods for a class of partially axisymmetric problems: application to the scattering of acoustic waves, PhD thesis, University of Colorado, 2002.

[9] F. Ihlenburg, Finite Element Analysis of Acoustic Scattering, Springer-Verlag, New York, 1998.

[10] F. Ihlenburg and I. Babuška, Finite element solution of the Helmholtz equation with high wave number - Part I: the h-version of the FEM, Comput. Math. Appl., 30(9), 9-37, 1995.

[11] M. Melenk, On generalized finite element methods, PhD thesis, University of Maryland, 1995.

[12] F. Rellich, Darstellung der Eigenwerte von $\Delta u+\lambda u=0$ durch ein Randintegral, Math. Z., 46, 635-636, 1940. 